\section{Ciencia y tecnología en la Argentina 2015-2019: panorama del ajuste neoliberal}

Resumen: Se analizan las políticas implementadas para el sector de ciencia y tecnología durante el gobierno del Presidente Mauricio Macri, en el contexto del modelo de desarrollo neoliberal que ha impulsado su gestión, centrado en la exportación de materias primas sin elaborary en la valorización financiera. Tal modelo reformuló en los hechos la evolución del sector, paralizando proyectos en curso en áreas como defensa, satelital y nuclear, suspendiendo todo intento de impulsar el desarrollo de la industria tecnológica nacional desde el Estado. Se presentan datos e indicadores que dan cuenta de la magnitud de los recortes realizados a la inversión estatal en CyT, la pérdida del salario real de los profesionales del sector y el deterioro en las condiciones de trabajo en las actividades de I+D, en un marco general de ajuste y achicamiento del Estado. Palabras clave: ajuste CyT en Argentina, datos CyT Argentina, política científica en el gobierno de Macri, neoliberalismo en СуТ

Science and technology in Argentina 2015-2019: a view of the neoliberal adjustment

Abstract: The policies implemented for the science and technology sector during the government of President Mauricio Macri are analyzed, in the context of the neo-liberal development model that has driven its management. Focused on the export of raw materials and financial valorization, this model imposed a shift in the direction of the sector evolution. Ongoing projects in areas such as defense, satellital and nuclear development were paralyzed and attempts to boost the national technological industry were suspended. Data and indicators presented show the magnitude of the cuts made to the state investment in S\&T, the loss of the real wage of the sector professionals and the deterioration of the working conditions in the R\&D activities, in a general framework of structural adjustment and shrinking of the State. Keywords: CyT adjustment in Argentina, CyT Argentina data, scientific policy in the Macri government, neoliberalism in CyT

Ciência e tecnologia na Argentina 2015-2019: panorama do ajuste neoliberal

Resumo: As políticas implementadas para o setor de ciência e tecnologia durante o governo do presidente Mauricio Macri são analisadas, no contexto do modelo de desenvolvimento neoliberal que impulsionou sua gestão, focada na exportação de matérias-primas e na valorização financeira. Esse modelo reformulou de fato a evolução do setor, paralisando projetos em andamento em áreas como defesa, satélite e nuclear, suspendendo qualquer tentativa de impulsionar o desenvolvimento da indústria nacional de tecnologia do Estado. São apresentados dados e indicadores que mostram a magnitude dos cortes feitos no investimento estatal em C\&T, a perda do salário real dos profissionais do setor e a deterioração das condições de trabalho nas atividades de P\&D, em uma quadro geral de ajuste e encolhimento do Estado. Palavras-chave: Ajuste da CyT na Argentina, dados de CyT Argentina, política científica no governo Macri, neoliberalismo na C\&T

\section{Ciencia Tecnología y Política \\ Año 2 No 3 jul- dic 2019}

(⿸丆口

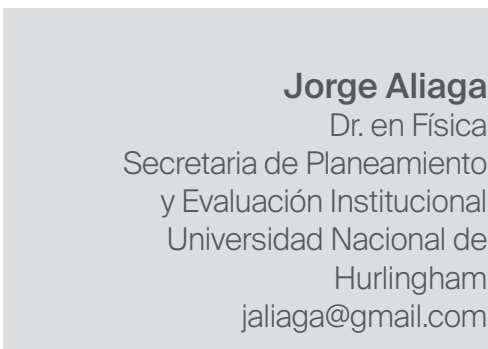

Año $2 \mathrm{~N}^{\circ} 3 \mathrm{Jul}$ - dic 2019 Fecha de recibido: 30/09/2019 Fecha de aprobado: 07/10/2019 https://doi.org/10.24215/26183188e024 https://revistas.unlp.edu.ar/CTyP ISSN 2618-3188

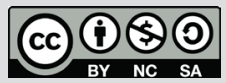

Esta obra está bajo licencia Creative Commons Atribución-NoComercial-Compartirlgual 4.0 Internacional http://creativecommons.org/licenses/bync-sa/4.O/deed.es_AR 


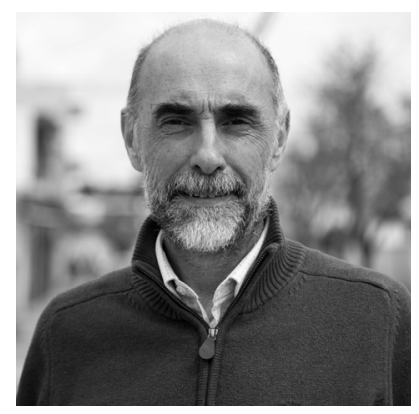

Jorge Aliaga

Dr. en Física

Secretaría de Planeamiento

y Evaluación Institucional

Universidad Nacional de

Hurlingham

jaliaga@gmail.com

\section{Ciencia y tecnología en la Argentina 2015-2019: panorama del ajuste neoliberal}

Resumen: Se analizan las políticas implementadas para el sector de ciencia y tecnología durante el gobierno del Presidente Mauricio Macri, en el contexto del modelo de desarrollo neoliberal que ha impulsado su gestión, centrado en la exportación de materias primas sin elaborar y en la valorización financiera. Tal modelo reformuló en los hechos la evolución del sector, paralizando proyectos en curso en áreas como defensa, satelital y nuclear, suspendiendo todo intento de impulsar el desarrollo de la industria tecnológica nacional desde el Estado. Se presentan datos e indicadores que dan cuenta de la magnitud de los recortes realizados a la inversión estatal en CyT, la pérdida del salario real de los profesionales del sector y el deterioro en las condiciones de trabajo en las actividades de I+D, en un marco general de ajuste y achicamiento del Estado.

\section{Introducción}

El actual gobierno nacional encontró al sector nacional de ciencia y tecnología (SNCyT) con un desarrollo de varias décadas. Los primeros cargos con dedicación exclusiva en las universidades nacionales fueron creados a principios de la década de 1960 por impulso de Rolando García, decano de la Facultad de Ciencias Exactas y Naturales de la Universidad de Buenos Aires. Para ese momento ya se habían creado varias instituciones del sector, relacionadas con sectores específicos como el agropecuario, nuclear, etc. Hoy el SNCyT cuenta con diversos organismos, que dependen de varios Ministerios y que forman parte del Consejo Interinstitucional de Ciencia y Tecnología (CICYT): 


\begin{tabular}{|c|c|c|}
\hline \multicolumn{3}{|c|}{ Consejo Interinstitucional de Ciencia y Tecnología (CICYT) } \\
\hline Instituciones integrantes & Sigla & Dependencia \\
\hline Consejo Nacional de Investigaciones Científicas y Técnicas & CONICET & Ministerio de \\
\hline Comisión Nacional de Actividades Espaciales & CONAE & $\begin{array}{l}\text { Cultura, Ciencia y } \\
\text { Tecnología }\end{array}$ \\
\hline Consejo Interuniversitario Nacional & $\mathrm{CIN}$ & $\begin{array}{l}\text { Universidades } \\
\text { Públicas }\end{array}$ \\
\hline Consejo de Rectores de Universidades Privadas & CRUP & $\begin{array}{l}\text { Universidades } \\
\text { Privadas }\end{array}$ \\
\hline Instituto Nacional de Tecnología Agropecuaria & INTA & \multirow{3}{*}{$\begin{array}{c}\text { Ministerio } \\
\text { de la Producción } \\
\text { y el trabajo }\end{array}$} \\
\hline Instituto Nacional de Tecnología Industrial & INTI & \\
\hline Instituto Nacional de Desarrollo Pesquero & INIDEP & \\
\hline Comisión Nacional de Energía Atómica & CNEA & \multirow{3}{*}{$\begin{array}{l}\text { Ministerio de } \\
\text { Hacienda }\end{array}$} \\
\hline Servicio Geológico Minero Argentino & SEGEMAR & \\
\hline Instituto Nacional del Agua & INA & \\
\hline $\begin{array}{l}\text { Instituto de Investigaciones Científicas y Técnicas para la } \\
\text { Defensa }\end{array}$ & CITEDEF & \multirow{4}{*}{$\begin{array}{l}\text { Ministerio } \\
\text { de Defensa }\end{array}$} \\
\hline Instituto Geográfico Nacional & IGN & \\
\hline Servicio de Hidrografía Naval & $\mathrm{SHN}$ & \\
\hline Servicio Meteorológico Nacional & SMN & \\
\hline Administración Nacional de Laboratorios e Institutos de Salud & ANLIS & $\begin{array}{c}\text { Ministerio } \\
\text { de Salud y Desarrollo } \\
\text { Social }\end{array}$ \\
\hline Instituto Antártico Argentino & IAA & $\begin{array}{c}\text { Ministerio } \\
\text { de Relaciones } \\
\text { Exteriores y culto }\end{array}$ \\
\hline
\end{tabular}

Desde el punto de vista presupuestario, el mo centralizado dependiente del MinisteCRUP no tiene una asignación específica, rio del Interior, Obras Públicas y Vivienda. si bien las universidades privadas alojan en sus ámbitos investigadores del CONICET que asimismo reciben financiamiento de entidades públicas. El INIDEP depende presupuestariamente de la finalidad Servicios Económicos, función Agricultura y el SMN de la misma finalidad, pero función Transporte. El resto de los organismos conforman la función Ciencia y Técnica dentro de la finalidad Servicios Sociales conjuntamente con la Fundación Miguel Lillo, la Secretaría de Gobierno de Ciencia y Tecnología (ex - MinCyT) y el Instituto Nacional de Prevención Sísmica (INPRES), organis-

En la campaña electoral presidencial del año 2015 numerosos miembros del sector CyT manifestaron preocupación por las políticas que podía implementar el candidato Mauricio Macri en caso de ganar las elecciones. Ante esa situación, y con la intención de llevar tranquilidad, Macri propuso primero elevar la inversión en Ciencia y Técnica al 1,5\% del PBI y, luego de ganar el ballotage, anunció la continuidad del entonces ministro del área, Lino Barañao. Habiendo casi cumplido los cuatro años de la actual gestión es posible realizar una evaluación de las políticas implementadas. 


\section{EI SNCyT en 2015}

Para el año 2015 el SNCyT había salido de la situación crítica en la que estaba luego de la crisis del 2001. Las políticas de incremento de becas doctorales del CONICET, que pasaron de 400 en 2002 a 1.200 en 2004, produjeron una suba de la cantidad de egresados en las carreras de doctorado. Los 500 potenciales nuevos investigadores, egresados de carreras de doctorado de todo el país en el año 2002 pasaron a ser 2.400 en el año 2015. El CONICET triplicó su planta de investigadores, pasando de 3.800 en 2003 a más de 9.200 en 2015. Los cargos de dedicación exclusiva en las universidades pasaron de 15.160 en 2003 a 21.144 en 2015. También se observó un incremento en el personal del resto de los organismos del SNCYT, si bien no hubo una coordinación en relación con la formación de jóvenes investigadores y su inserción en el sistema, como debió ocurrir a través del CICYT. Como excepción vale destacar el acuerdo alcanzado por el sector en el año 2012 en aceptar a los Proyectos de DesarroIlo Tecnológico y Social (PDTS) como mecanismo de evaluación del personal del SNCyT alternativo a la producción científica medida en términos de la cantidad de trabajos publicados o patentes. En ese período hubo un desarrollo de algunas iniciativas de impulso a la industria tecnológica nacional mediante el uso del poder de compra estatal. La decisión de finalizar con la construcción de Atucha II, que había sido abandonada en la década de 1990, significó el resurgimiento de pequeñas y medianas empresas (PYMES) relacionadas con el área nuclear. INVAP pasó de tener 300 empleados en 2003 a casi 1.500 en 2015 por la decisión del gobierno nacional de construir en el país radares para la aeronavegación, la defensa y meteorológicos, así como satélites de observación y de comunicaciones. Esto implicó la creación de decenas de pequeñas empresas tecnológicas en Bariloche, Córdoba y La Plata. A partir del año 2014 se creó la Secretaría de Ciencia, Tec- nología y Producción para la Defensa, que agrupó a los organismos del SNCYT del área (CITEDEF, SHN, SMN, IGN) con las fábricas militares: Dirección Nacional de Fabricaciones Militares (FFMM), Fábrica Argentina de Aviones (FAdeA) y el Complejo Industrial y Naval Argentino (CINAR). Esa Secretaría lanzó el Programa de Investigación y Desarrollo para la Defensa (PIDDEF) mediante el cual financió proyectos de investigación aplicados a temas de interés del Ministerio de Defensa. También, luego de la recuperación por parte del Estado nacional de la mayoría accionaria de Yacimientos Petrolíferos Fiscales (YPF), se decidió la creación de YPF Tecnología S.A. (Y-TEC), empresa conformada en un 51\% por YPF y en un 49\% por CONICET, con el objetivo de generar soluciones tecnológicas para el desarrollo energético.

\section{EI SNCyT desde el 10/12/2015}

Desde la asunción del gobierno de Mauricio Macri el autor de este trabajo fue recopilando las noticias relacionadas con el SNCYT que se difundían por los medios (Aliaga, 2019). El repaso de esas páginas permite recuperar la perspectiva de los cambios que ocurrieron. Una de las primeras definiciones concretas sobre las nuevas políticas que iba a aplicar el nuevo gobierno fue explicitada el 23 de febrero del año 2016 cuando el entonces Secretario de Comercio Miguel Braun visitó el Atlantic Council de Nueva York (Braun, 2016). Braun indicó que se estaba ante una "nueva Argentina" y que ésta iba a dedicarse a las cosas en las que era "naturalmente competitiva", entendiendo esto último como aquéllas en las que se era competitivo dejando que el mercado actúe sin la intervención del Estado. El listado se limitaba a la agroindustria, la minería, las energías alternativas, la energía no-convencional, los productos audiovisuales y el software. Según ese diagnóstico, el resto de los sectores debían reconvertirse implicando en los hechos la desaparición de la mayoría de las PYMES 
nacionales. Esta clara definición del modelo a seguir reformuló en los hechos la evolución del SNCYT. Se suspendió todo intento de impulsar el desarrollo de la industria tecnológica nacional desde el Estado. En febrero de 2016 el Ministerio de Defensa anuló el convenio firmado con INVAP un año antes por dos mil millones de pesos para desarrollar el Sistema Aéreo Robótico Argentino (SARA) (De la Vega, 2016). Esta iniciativa se había iniciado en el año 2010 e INVAP ya había elaborado un prototipo. También el Ministerio de Defensa despidió personal de Fabricaciones Militares y suspendió diversos proyectos, incluyendo el de construcción de vagones ferroviarios. En paralelo el Ministerio de Seguridad inició la compra de radares y drones a Israel. También inmediatamente luego de asumir la nueva gestión de ARSAT (Empresa Argentina de Soluciones Satelitales Sociedad Anónima) suspendió la construcción del ARSAT 3, que debía ser lanzado en el año 2019 para dar servicios de banda Ka. Simultáneamente se autorizó que dieran servicio en el país satélites de empresas extranjeras, limitando las posibilidades de que la empresa nacional mantuviera condiciones comerciales adecuadas dentro del mercado nacional (Krakowiak, 2018). La suspensión de proyectos que el Estado contrataba a INVAP se vio acompañado de una demora en el pago de los contratos vigentes. Con el objetivo de lograr llevar a cero el déficit fiscal primario, el gobierno nacional no solamente achicó presupuesto y postergó el devengado de los mismos sino que también atrasó los pagos (Bermúdez, 2019). En el caso de INVAP esto generó severas complicaciones dadas las dificultades para obtener financiamiento bancario a tasas de interés razonables (Latam Satelital, 2018). El gobierno lanzó planes para el desarrollo de proyectos de generación de energías alternativas, fundamentalmente eólica y solar. Pero en lugar de establecer condiciones que impulsaran la construcción de al menos una parte tecnológicamente relevante en el país favoreció la maximización de ingresos de las generadoras. La normativa permitió la importación de equipos usados, obteniendo una exención impositiva total si se fabricaban partes menores en el país, como por ejemplo las cabinas metálicas de los molinos eólicos (Hurtado, 2018). En el caso del INTA, se desfinanciaron los centros de extensionismo rural y el apoyo a la agricultura familiar y se impulsó la reducción de la planta de empleados. En el INTI directamente despidieron 250 trabajadores científicos y tecnológicos contratados y al mismo tiempo se posibilitó mediante el cambio de la normativa vigente que empresas privadas brindaran el servicio de certificación que era exclusivo de esa institución.

\section{La situación del sector nuclear}

La situación en el sector nuclear es mucho más preocupante, dado el desarrollo que alcanzó éste luego de setenta años de planificación e inversión estatal. El anterior gobierno había acordado con China el financiamiento para la construcción de dos centrales de potencia, la primera con tecnología Candu, usando uranio natural y agua pesada, y la segunda de tecnología China de uranio enriquecido y agua liviana. Mientras que la venta de la segunda era de interés de China, el cierre del ciclo de tecnología Candu de la primera central a construirse era estratégico dentro del plan histórico de desarrollo nacional del sector. El gobierno anuló los convenios argumentando que eran muy costosos y finalmente firmó uno mediante el cual solamente se construirá la central que China está interesada en vender. Esto deja sin continuidad a decenas de PYMEs nucleares nacionales y al borde del cierre a la Planta Industrial de Agua Pesada (PIAP), situada en Arroyito, Neuquén. La PIAP ha reducido su personal en estos años y los empleados que no se han retirado sufren demoras en el cobro de los salarios. Asimismo se ha desfinanciado a DIOXITEC, la empresa encargada de fabricar las celdas de combustible nuclear para 
| Ciencia, Tecnología y Política | Año 2 | ํo3 | Julio-Diciembre 2019 | ISSN 2618-3188 | www.revistas.un|p.edu.ar/CTyP |

las centrales de potencia nacionales (Rocca, 2019).

\section{La situación del CONICET y las Universidades Nacionales (UUNN)}

Finalmente analicemos la situación del CONICET y de las UUNN, que concentran la mayor parte del personal del SNCyT. Por el lado del CONICET, la cantidad de becas otorgadas por año para realizar doctorados volvió a los valores del período 2004-2012, de aproximadamente 1.200, frente a las 1.500 que se habían otorgado entre los años 2013 y 2015. El ingreso a carrera del investigador fue el que sufrió el mayor recorte. Entre los años 2010 y 2015 la cantidad de ingresos anuales pasó de 600 a 950, con un incremento de aproximadamente $10 \%$ anual. En el año 2016 ese número bajó a 502, de los cuales 345 fueron en temas generales, 40 en temas estratégicos, 70 desde el exterior y 47 reconsideraciones. Ese año se conoció además que aproximadamente 500 postulantes habían sido recomendados para ingresar tanto por la evaluación de la comisión disciplinar como la de junta, y no habían entrado por falta de fondos. Ante el reclamo, que incluyó movilizaciones y la toma del MinCyT el gobierno lanzó, por única vez, el "Programa de Incorporación de Docentes Investigadores a las Universidades Nacionales". Luego de un año de trámites, aproximadamente 440 de los llamados "dobles recomendados" 2016 obtuvieron cargos interinos de dedicación exclusiva en UUNN. Para el año 2017 el CONICET avisó que habría 600 ingresos, 300 de los cuales serían en temas generales y los otros 300 en tecnología y temas estratégicos. Además eliminó la posibilidad de presentarse desde el exterior. En la convocatoria 2018 el CONICET volvió a cambiar las reglas, pasando a 150 ingresos generales, 150 en tecnología y temas estratégicos y 150 para "fortalecimiento" de instituciones del SNCYT con menos de 120 investigadores del CONICET. La otra novedad fue que los resulta- dos de la convocatoria anual por primera vez no se conocieron entre los meses de noviembre y diciembre del año correspondiente sino en el mes de abril del año siguiente, generando un diferimiento en el gasto para el siguiente ejercicio presupuestario. La convocatoria 2019 se llamó igual a la del año 2018, aunque se verá cuando se conozcan los resultados, en abril de 2020, si el gobierno de ese momento incorpora vacantes adicionales (Piscetta, 2019).

\section{Recortes de presupuesto y caída de salarios}

Además el SNCyT tuvo dificultades para funcionar como consecuencia de la demora en el pago de subsidios y la pérdida de valor del peso como resultado de las sucesivas devaluaciones. Se recortó el presupuesto de la Agencia Nacional de Promoción Científica y Tecnológica (ANPCyT), especialmente la componente financiada con recursos del tesoro nacional. En el caso de los institutos del CONICET, el financiamiento de las unidades ejecutoras se vio muy limitado dado que el presupuesto de funcionamiento del CONICET pasó de ser el 10\% del total en 2015 a menos del 5\% en 2018. Adicionalmente a los ajustes antes descriptos hay que destacar que en el año 2016 y fundamentalmente en 2018 y 2019 se produjo una importante pérdida salarial, igual a la del resto de los empleados estatales (Ballarino, 2019). Para comparar la inversión del SNCYT se pueden tomar los datos de Indicadores publicados por la Dirección Nacional de Información Científica dependiente de la Subsecretaría de Estudios y Prospectiva de la Secretaría de Planeamiento y Políticas de la Secretaría de Gobierno de Ciencia, Tecnología e Innovación Productiva (ex - MinCyT). La Figura 1 muestra los datos de Inversión en CyT en términos del Producto Bruto Interno (PBI) publicados en marzo del 2019 (Indicadores, 2019). Los últimos datos dis- 
| Ciencia, Tecnología y Política | Año 2 | N³ | Julio-Diciembre 2019 | ISSN 2618-3188 | www.revistas.unlp.edu.ar/CTyP |

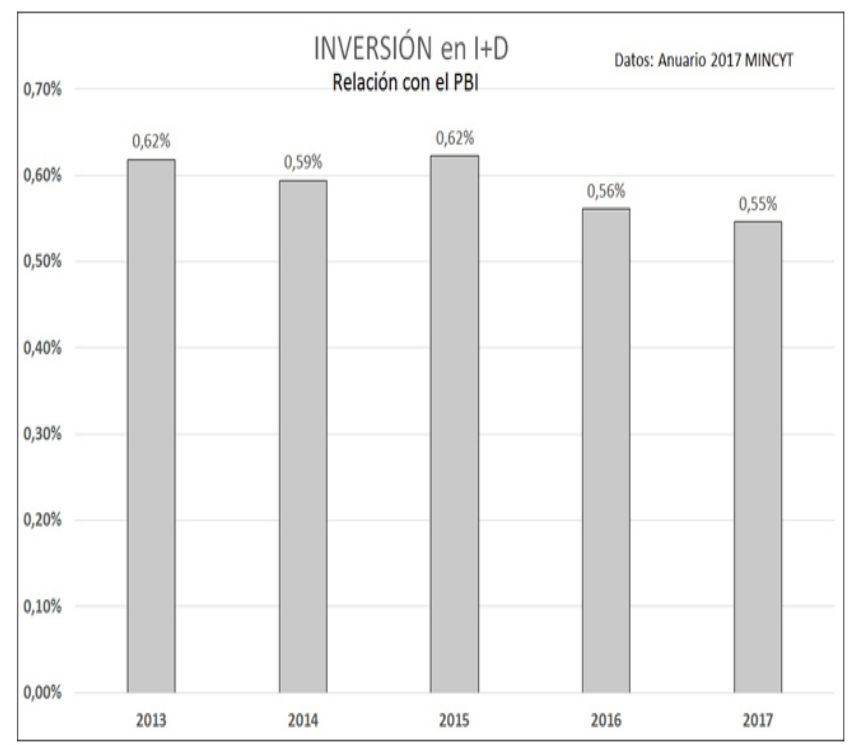

Figura 1: Inversión en CyT en Argentina en términos del Producto Bruto Interno (PBI). Fuente: Secretaría de Gobierno de Ciencia, Tecnología e Innovación Productiva (Indicadores, 2019). Marzo 2019.

ponibles corresponden al año 2017, por lo que no se refleja todavía el recorte producido en 2018 y 2019. Según los datos publicados por el ex- MinCyT, aproximadamente el 50\% de la inversión en CyT corresponde al financiamiento del gobierno nacional, mientras que el resto corresponde en partes iguales a empresas y a la fracción de los salarios de los docentes con dedicación exclusiva que se destina a tareas de I+D. Cabe aclarar que desde el punto de vista presupuestario los salarios docentes figuran dentro de la función Educación. Por lo tanto, la función Ciencia y Técnica del presupuesto nacional puede usarse como un estimador de la inversión en CyT si se la multiplica por un factor dos. La ventaja de tomar el presupuesto es que, a diferencia del dato que elabora el ex - MinCyT, se conoce a medida que transcurre el año y no con dos años de demora. La Figura 2 muestra el presupuesto ejecutado para la Función CyT en términos del Producto Bruto Inter-

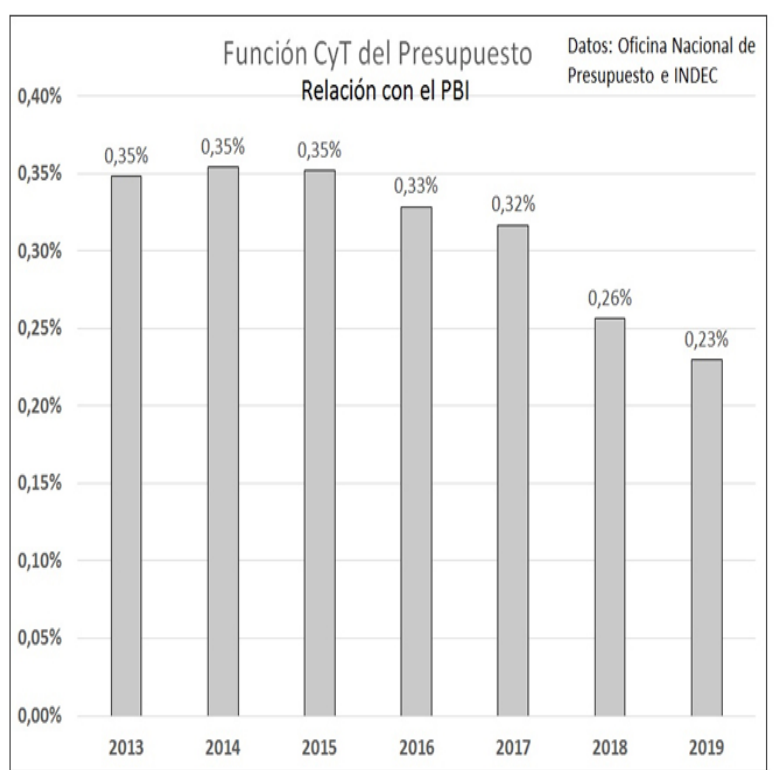

Figura 2: Función CyT en términos del Producto Bruto Interno (PBI). El dato del PBI 2019 fue el estimado en el presupuesto del año, presentado en septiembre de 2018. Para los años 20132018 se tomó el devengado o ejecutado, que consiste en la obligación de pago que surge cuando se reciben los bienes o servicios pactados. Para el año 2019 se tomó el presupuesto vigente al 31 de julio del 2019.

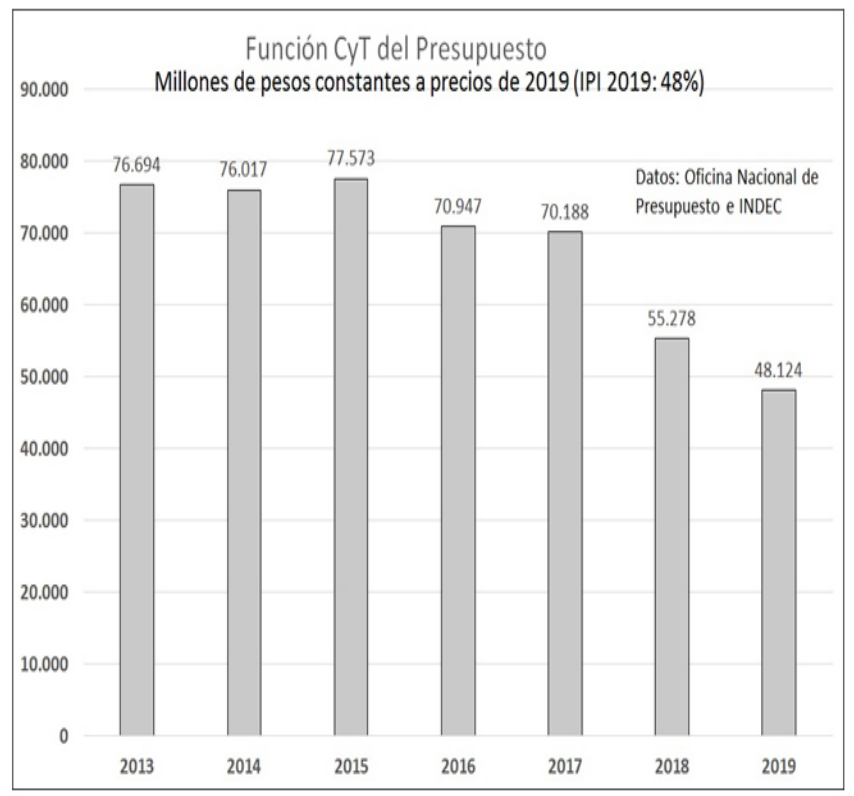

Figura 3: Función CyT en millones de pesos de 2019. ${ }^{2}$

\footnotetext{
${ }^{1}$ Los datos de ejecución presupuestaria son publicados en el sitio oficial de la Oficina Nacional de Presupuesto (ONP, 2019). Los datos del PBI y del Índice de Precios Implícitos (IPI), que se usa tanto para realizar comparaciones interanuales entre PBI como de presupuestos, son publicados por el Instituto Nacional de Estadísticas y Censos (INDEC) (INDEC-PBI, 2019).

2 Para realizar el ajuste interanual se utilizó el Índice de Precios Implícitos (IPI) con base 100 para el año 2004, publicado por la actual gestión del INDEC (INDEC-PBI, 2019, Cuadro 13). El IPI es un índice calculado con promedios de variación. Para convertir los pesos de cada año al valor del año 2019 se tomaron los siguientes valores: IPI(2013) = 464,78; IPI(2014) = 652,01; IPI(2015) = 825,31; IPI(2016) = 1164,67; IPI(2017) = 1467,58; IPI(2018) = 2064,92; IPI(2019) = 3056,08. Con el valor tomado en el presupuesto 2020 el IPI(2019) = 3159,33.
} 
no (PBI). La Figura 3 muestra los datos de la Función CyT en millones de pesos del año 2019. ${ }^{2}$ Para el año 2019 se tomó el valor de inflación interanual minorista (IPC) del 40\% que se estimaba en julio de 2019. Con ese valor de IPC, al 31 de julio de 2019 el IPI ascendía al $48 \%$ y el valor del dólar era de $\$ 45$. Con posterioridad se produjo una devaluación del 30\%, lo que hará que la inflación interanual minorista del año 2019 sea superior al 50\%. Como referencia, en el presupuesto presentado para el año 2020 se estima para el año 2019 un IPC del 52,8\% y un IPI del 53,0\%, cuando al presentar ese presupuesto en septiembre de 2018 se habían tomado valores del 23,0\% y 34,8\%, respectivamente. Sólo si hasta fin de año se incorporan partidas adicionales, parte de este deterioro adicional producido con posterioridad al 31 de julio de 2019 se compensará, al menos parcialmente.

La Tabla 1 muestra el devengado para cada orga- nismo, en el caso de los que tienen presupuesto descentralizado, o ministerio, para el caso de organismos con presupuesto centralizado, para el año 2015, tanto en millones de pesos de ese año como de pesos de 2019. También se muestran los presupuestos de 2019 al 31 de julio, la diferencia entre el mismo y el devengado en 2015 en pesos de 2019 y la diferencia porcentual. El ajuste en la función CyT entre 2015 y 2019 llega al 38\%, tomando los créditos vigentes al 31 de julio y la inflación que se estimaba en ese momento. Si se analiza la variación por objetivo se observa una baja en el "Inciso 1: Gastos en Personal" del orden del 30\%, salvo en el CONICET donde la disminución es menor porque la planta se incrementó aproximadamente un 15\% desde 2015. En CONICET los Gastos en Personal, que cubren los salarios de los investigadores, técnicos y personal administrativo, registró una

\begin{tabular}{|l|l|l|l|l|l|}
\hline & 2015 & 2019 & & \\
\hline & $\begin{array}{l}\text { Millones de } \\
\$ \text { de } 2015\end{array}$ & $\begin{array}{l}\text { Millones de } \\
\$ \text { de } 2019^{3}\end{array}$ & $\begin{array}{l}\text { Millones de } \$ \\
\text { de } 2019\end{array}$ & Diferencia & $\begin{array}{l}\% \\
\text { Diferencia }\end{array}$ \\
\hline 101 - Fundación Miguel Lillo & $\$ 126,21$ & $\$ 467,35$ & $\$ 316,27$ & $\$-151,08$ & $-32,33 \%$ \\
\hline 103 - CONICET & $\$ 6.338,73$ & $\$ 23.471,98$ & $\$ 16.965,92$ & $\$-6.506,06$ & $-27,72 \%$ \\
\hline 105 - CNEA & $\$ 2.871,04$ & $\$ 10.631,31$ & $\$ 7.108,22$ & $\$-3.523,09$ & $-33,14 \%$ \\
\hline 106 - CONAE & $\$ 1.833,24$ & $\$ 6.788,39$ & $\$ 1.951,73$ & $\$-4.836,66$ & $-71,25 \%$ \\
\hline 108 - INA & $\$ 157,77$ & $\$ 584,21$ & $\$ 327,71$ & $\$-256,51$ & $-43,91 \%$ \\
\hline 336 - \$GCXT & $\$ 2.635,28$ & $\$ 9.758,30$ & $\$ 4.517,24$ & $\$-5.241,06$ & $-53,71 \%$ \\
\hline 450 - IGN & $\$ 103,45$ & $\$ 383,07$ & $\$ 205,10$ & $\$-177,97$ & $-46,46 \%$ \\
\hline 606 - INTA & $\$ 3.667,18$ & $\$ 13.579,37$ & $\$ 7.785,94$ & $\$-5.793,44$ & $-42,66 \%$ \\
\hline 608 - INTI & $\$ 1.146,78$ & $\$ 4.246,45$ & $\$ 2.215,39$ & $\$-2.031,06$ & $-47,83 \%$ \\
\hline 624 - SEGEMAR & $\$ 200,65$ & $\$ 743,00$ & $\$ 444,50$ & $\$-298,50$ & $-40,17 \%$ \\
\hline 906 - ANLIS & $\$ 434,37$ & $\$ 1.608,45$ & $\$ 1.012,23$ & $\$-596,22$ & $-37,07 \%$ \\
\hline Min DEFENSA (SHN, CITEDEF, etc) & $\$ 970,16$ & $\$ 3.592,46$ & $\$ 1.346,59$ & $\$-2.245,87$ & $-62,52 \%$ \\
\hline Min EDUCACION (UUNN) & $\$ 313,84$ & $\$ 1.162,12$ & $\$ 1.385,91$ & $\$ 223,80$ & $19,26 \%$ \\
\hline Min INTERIOR (INPRES) & $\$ 36,98$ & $\$ 136,94$ & $\$ 60,79$ & $\$-76,15$ & $-55,61 \%$ \\
\hline Min REL EXT (IAA) & $\$ 113,37$ & $\$ 419,79$ & $\$ 285,68$ & $\$-134,12$ & $-31,95 \%$ \\
\hline $\begin{array}{l}\text { Banco Nacional de Datos Genéticos } \\
\text { (BNDG) }\end{array}$ & $\$ 0,00$ & $\$ 0,00$ & $\$ 5,40$ & $\$ 5,40$ & \\
\hline $\begin{array}{l}\text { OBLIGACIONES A CARGO DEL } \\
\text { TESORO }\end{array}$ & $\$ 0,00$ & $\$ 0,00$ & $\$ 2.219,11$ & $\$ 2.219,11$ & \\
\hline TOTAL & $\$ 20.949,04$ & $\$ 77.573,18$ & $\$ 48.153,70$ & $\$-29.419,48$ & $-37,92 \%$ \\
\hline
\end{tabular}

Tabla 1: Presupuestos devengados en 2015 en millones de pesos de 2015 y 2019 y presupuesto al 31/07 de 2019. Las diferencias absolutas y relativas corresponden a los montos en pesos 2019 devengados en 2019 y 2015.

\footnotetext{
${ }^{3}$ La conversión a pesos de 2019 se hizo usando el IPI(2919) = 3056,08 vigente al 31 de julio.
} 
baja del 20,2\% entre 2015 y 2019. Los montos asignados a Servicios No Personales, que corresponden mayoritariamente a las Becas, tiene una disminución del 35,2\% en el mismo período. Finalmente los montos asignados a Gastos de Funcionamiento tienen una baja del 56.5\%. La ANPCyT, cuyo presupuesto corresponde al Programa 44 de la SGCYT, tiene una caída entre los años 2015 y 2019 del 40,7\%, teniendo el financiamiento de fuentes externas correspondientes a créditos del Banco Interamericano de Desarrollo (BID) y del Banco de Desarrollo de América Latina (CAF) una baja del $27,5 \%$ y el financiamiento del tesoro nacional una caída del $62,4 \%$. Los programas 1 y 43 de la SGCT, que incluyen al Consejo Federal del Ciencia y Tecnología (COFECyT), planeamiento, evaluación y articulación del sistema científico, sistemas nacionales, biblioteca electrónica y el Centro Cultural de la Ciencia (C3) registran una baja del 67,9\% entre 2015 y 2019. Para coronar el proceso de ajuste y degradación de la situación del SNCYT en septiembre de 2018 el gobierno eliminó el Ministerio de Ciencia, Tecnología e Innovación Productiva (MinCyT) y lo transformó en una Secretaría de Gobierno dependiente del Ministerio de Educación (Bär, 2018). En los hechos el cambio no significó un ajuste adicional sino una desjerarquización simbólica y la pérdida del lugar específico en las reuniones de gabinete. La función CyT del Presupuesto de las UUNN ha sido la única que se ha incrementado desde 2015. Ésta se destina a Becas y Subsidios de esas instituciones y a los programas de incentivos y fortalecimiento de la Secretaría de Políticas Universitarias (SPU). Cabe destacar que el incremento se asignó a programas de fortalecimiento manejados desde la SPU y no directamente a las UUNN. Estos programas han registrado demoras en su ejecución y también en el pago. Como ejemplo, en el año 2018 el presupuesto fue de \$ 1.348,57 millones, se devengaron \$910,01 millones y solamente se pagaron $\$ 295,07$ millones.

\section{Conclusiones}

El actual gobierno ha intentado instalar definitivamente en el país un modelo basado en la exportación de materias primas sin elaborar y en la valorización financiera. No es de extrañar entonces que se haya achicado significativamente la inversión en CyT, a la par que se ajustaba la inversión y el gasto del Estado. Los salarios de los investigadores se han deteriorado severamente y los subsidios para la investigación se encuentran tan devaluados que es imposible mantener la actividad de los laboratorios y centros. Vale mencionar que los datos presentados se calcularon tomando en cuenta el IPI, que se usa para ajustar presupuestos y PBI. Según este, entre los años 2015 y 2019 los pesos se reajustaron por un factor de 3,69 veces, resultante de dividir el IPI del año 2019 por el del año 2015. Ese valor sube a 3,83 veces si se usa el valor estimado para 2019 en el presupuesto 2020. Sin embargo, en ese período, al 31 de julio de 2019, el peso se había devaluado en un factor 4,5 en término de dólares, y esta relación aumentó sustancialmente luego de esa fecha. Medido en dólares la caída de la economía Argentina en este período es mucho mayor. En el año 2015 el PBI era de \$ 5.954 .511 millones, lo que equivalía a U\$S 595.451 millones. En el presupuesto presentado para el año 2020 se estima un PBI para el año 2019 de $\$ 21.780 .498$ millones, lo que equivale a U\$S 454.803 millones tomando el valor promedio del dólar de \$47,89 usado allí. Es decir que la función CyT del Presupuesto nacional era el 0,352\% de PBI de ese año y bajó a un 0,215\% de un PBI que es 23,4\% menor. Entre 2015 y 2019 se pasó de un presupuesto que equivalía a U\$S 2.096 millones a uno que equivale a U\$S 978 millones. Por lo tanto, recuperar la inversión en dólares que había en el año 2015 implicaría un aumento muy superior al que se 
refleja en la caída del 38\% en pesos, que se obtiene considerando la evolución del IPI. En un contexto de gran endeudamiento externo y de extremas necesidades del conjunto de la población, que se refleja en el aumento de la pobreza y la indigencia, el gobierno que asuma en 10 de diciembre de 2019 deberá tomar medidas urgentes que atenúen la crisis actual del sistema de CyT. En paralelo se deberán planificar acciones de mediano y largo plazo que contribuyan a relanzar, mediante el poder de compra del Estado, sectores industriales estratégicos que se apoyen en las capacidades científicas y tecnológicas con las que todavía cuenta el país.

\section{Bibliografía}

Aliaga, J. (2019). Observatorio de CyT. Recuperado de http://www.jorgealiaga.com.ar/?page_id=1252

Ballarino, F. (23 de mayo de 2019). Directores del Conicet realizaron un cabildo abierto en defensa de la ciencia. Perfil. Recuperado de https://www.perfil. com/noticias/ciencia/directores-del-conicet-reali zaron-un-cabildo-abierto-en-defensa-de-la-cien cia-argentina.phtml

Bär, N. (3 de septiembre de 2018). Rechazo y pesar entre los investigadores por la eliminación del Ministerio de Ciencia. La Nación. Recuperado de https://www.lanacion.com.ar/sociedad/rechazo-pesar-investigadores-eliminacion-del-ministe rio-ciencia-nid2168470

Bermúdez, E. (24 de enero de 2019). Según cómo se calcule, el déficit fue mayor al anunciado. Clarín. Recuperado de https://www.clarin.com/economia/ economia/calcule-deficit-mayor-anunciado_O_ 497FKNx92.html

Braun, M. (23 de febrero de 2016). Conversation with Argentina Secretary of Trade: New Government, New Outlook. Atlantic Council. Recuperado de https://youtu.be/7weyz-Mc6el

DelaVega,M.(30demayode2016).FuegoamigocontraelSARA. Agencia TSS. Recuperado dehttp://www. unsam.edu.ar/tss/fuego-amigo-contra-el-sara/ Hurtado, D. (15 de abril de 2018). ENERGÍAS RENOVABLES: ¿NEGOCIO FINANCIERO O POLÍTICA INDUSTRIAL? El Cohete a la Luna. Recuperado de https://www.elcohetealaluna.com/energias-reno vables-negocio-financiero-o-politica-industrial/

INDEC-PBI, (19 de junio de 2019). Agregados macro-económicos (PIB). INDEC. Recuperado de https://www.indec.gob.ar/ftp/cuadros/economia/ sh_oferta_demanda_06_19.xls

Indicadores, (16 de abril de 2019). Indicadores de Ciencia y Tecnología Argentina 2017. Secretaría de Gobierno de Ciencia, Tecnología e Innovación Productiva. Recuperado de https://www.argentina.gob. ar/sites/default/files/indicadores_de_cyt._argenti na_2017_2.xlsx

Krakowiak, F. (12 de febrero de 2018). Cielos abiertos para las corporaciones globales. Página 12. Recuperado de https://www.pagina12.com.ar/95189-cie los-abiertos-para-las-corporaciones-globales

Latam Satelital (1 de diciembre de 2018). Informe de INVAP sobre contratos con el Estado Nacional. Latam Satelital. Recuperado de http://latamsatelital. com/informe-invap-contratos-estado-nacional/

ONP (2019). Presupuesto Abierto, Oficina Nacional de Presupuesto. Ministerio de Hacienda. Recuperado de https://www.presupuestoabierto.gob.ar/ sici/

Piscetta, J. (6 de abril de 2019). Revuelo en el ámbito científico: más de 2 mil investigadores fueron excluidos del Conicet. Infobae. Recuperado de ht tps://www.infobae.com/sociedad/2019/04/06/re vuelo-en-el-ambito-cientifico-mas-de-2-mil-inves tigadores-fueron-excluidos-del-conicet/

Rocca, G. (3 de julio de 2019). Un desastre atómico. Nex Ciencia. Recuperado de https://nexcien cia.exactas.uba.ar/programa-nuclear-argentino-pe ligro-ajuste-cuarta-central-candu-cnea-an dres-kreiner 